\title{
Ofatumumab use in Juvenile Systemic Lupus Erythematosus: A Single Centre Experience
}

Authors: Ovgu Kul Cinar ${ }^{1}$, Matko Marlais ${ }^{2}$, Muthana Al Obaidi ${ }^{1}$, Fan lek Cheng ${ }^{3}$, Kjell Tullus ${ }^{2}$ Paul Brogan ${ }^{1,4 *}$, Elena Moraitis ${ }^{1 *}$

\section{Affiliations:}

1 Paediatric Rheumatology Department, Great Ormond Street Hospital, London, UK

2 Paediatric Nephrology Department, Great Ormond Street Hospital, London, UK

3 Pharmacy, Great Ormond Street Hospital, London, UK

4 Infection, Immunity and Rheumatology Section, Great Ormond Street Institute of Child Health, London, UK

*These authors contributed equally to the manuscript

Word Count: 498

Rituximab, a chimeric monoclonal antibody that specifically targets CD20-positive B cells, is an evolving therapy which has been used in refractory juvenile systemic lupus erythematosus (SLE). However, infusion reactions are common and can prevent the use for repeat treatment in patients who demonstrated beneficial response $(1,2)$.

Ofatumumab is a fully humanized anti-CD20 monoclonal antibody (mAb) which has been licensed for use in haematological malignancies, rheumatoid arthritis and paediatric nephrotic syndrome $(3,4)$. There are limited data on the off-label use of Ofatumumab as an alternative B-cell depletion agent for patients with systemic lupus erythematosus (SLE) allergic to Rituximab, with minimal data on its use in juvenile SLE (jSLE patients) $(3,5)$. Therefore, we aim to describe single-centre retrospective case series of patients treated with off-label ofatumumab for jSLE between June 2018-April 2020 at Great Ormond Street Hospital, UK. Demographics, clinical and laboratory characteristics and treatment were collected (Table 1).

Three patients were identified: 3/3 females (1/3 Afro-Caribbean, 2/3 Asian, with median age 14 years (range:12-16 years), and median jSLE disease duration: 31 months (range: 16-71 months). All three patients received Rituximab, Mycophenolate Mofetil (MMF) and steroids 
prior to Ofatumumab. In addition, 2/3 patients had intravenous cyclophosphamide (cases 1 and 2). Post-Ofatumumab, all patients remained on MMF maintenance therapy, and weaning course of steroids.

The indication for ofatumumab in 3 patients was active jSLE, with severe prior reaction to Rituximab. The median number of Rituximab infusions received was 2 (range 1-4). The median duration between the last Rituximab dose and the first Ofatumumab dose was 9 months (range 4 days-55 months).

Active organs/systems involved prior to ofatumumab were: neurological and renal involvement for case 1; haematological involvement and serositis for case 2; and haematological involvement (ITP and immune haemolytic anaemia) for case 3. Cases 1 and 3 received one course of Ofatumumab ( $700 \mathrm{mg} /$ dose, 2 doses, administered on day 0 and day 14); case 2 received 2 courses, same dose, 9 months apart. The median follow up postOfatumumab was 14 months (range 8-23 months).

Significant clinical improvement was observed in all cases (Table 1), mirrored by improved laboratory markers of disease activity including anti-dsDNA antibody, complement levels, and proteinuria. At 6 months follow up, British Isles Lupus Assessment Index (BILAG) 2004 had improvement for all patients: $2 / 3$ from $A$ to $D ; 1 / 3$ from $B$ to $D$ as well as Systemic Lupus Erythematosus Disease Activity Index (SLEDAI). At 6 months follow-up, the disease remained well-controlled for $2 / 3$ patients, whereas $1 / 3$ patient had a disease flare 9 months after the Ofatumumab course and received a second course with good response. Lymphocyte subsets were only available for $2 / 3$ patients at 6 months post Ofatumumab. Two of the patients had repopulated $B$ cells at this time point. 
In this small series, treatment with Ofatumumab for patients with jSLE allergic to Rituximab was a safe, well-tolerated and effective alternative to Rituximab therapy for B cell depletion. The clinical and serological outcomes were favourable, and in our experience similar to those achieved after Rituximab.

References:

1. Terrier B, Amoura Z, Ravaud P et al. Safety and efficacy of rituximab in systemic lupus erythematosus: results from 136 patients from the French Autolmmunity and Rituximab registry. Arthritis Rheum 2010; 62: 2458-2466

2. Diaz-Lagares C, Croca S, Sangle $S$ et al. Efficacy of rituximab in 164 patients with biopsy-proven lupus nephritis: pooled data from European cohorts. Autoimmun Rev 2012; 11: 357-364

3. Masoud S, McAdoo SP, Bedi R, Cairns TD, Lightstone L. Ofatumumab for B cell depletion in patients with systemic lupus erythematosus who are allergic to rituximab. Rheumatology (Oxford). 2018;57(7):1156-1161.

4. Haarhaus ML, Svenungsson E, Gunnarsson I. Ofatumumab treatment in lupus nephritis patients. Clin Kidney J. 2016;9(4):552-555. doi:10.1093/ckj/sfw022

5. Lei L, Muhammad S, Al-Obaidi M, et al. Successful use of ofatumumab in two cases of early-onset juvenile SLE with thrombocytopenia caused by a mutation in protein kinase C $\delta$. Pediatr Rheumatol Online J. 2018;16(1):61. 

Case 1

Case 2

Case 3

\begin{tabular}{|c|c|c|c|c|c|c|}
\hline Ofatumumab & Pre & $\begin{array}{c}\text { Post } \\
\text { ( 6 months) }\end{array}$ & Pre & $\begin{array}{c}\text { Post } \\
\text { ( 6 months) }\end{array}$ & Pre & $\begin{array}{c}\text { Post } \\
\text { ( 6 months) }\end{array}$ \\
\hline Age, gender & & ears, $F$ & & ears, F & $12 y$ & months, $\mathrm{F}$ \\
\hline
\end{tabular}

\begin{tabular}{|c|c|c|c|}
\hline Ethnicity & Asian & Afro-Caribbean & Asian \\
\hline $\begin{array}{l}\text { Duration cSLE } \\
\text { (months) }\end{array}$ & 31 months & 71 months & 16 months \\
\hline $\begin{array}{l}\text { Previous } \\
\text { Therapies }\end{array}$ & $\begin{array}{c}\text { IVMP, prednisolone, } \\
\text { Rituximab, } \mathrm{HCQ}, \mathrm{MMF} \text {, } \\
\text { CYP }\end{array}$ & $\begin{array}{l}\text { IVMP, prednisolone, } \\
\text { Rituximab, AZA, HCQ, } \\
\text { MMF, CYP }\end{array}$ & $\begin{array}{l}\text { IVMP, IVIg, Rituximab, } \\
\text { prednisolone, HCQ, MMF }\end{array}$ \\
\hline $\begin{array}{l}\text { Previous total } \\
\text { Rituximab dose } \\
\text { (gram) }\end{array}$ & $\begin{array}{l}3 \text { grams-severe allergic } \\
\text { reaction ( } 4^{\text {th }} \text { dose) }\end{array}$ & $\begin{array}{l}1 \text { gram- severe allergic } \\
\text { reaction ( } 2^{\text {nd }} \text { dose) }\end{array}$ & $\begin{array}{c}1 \text { gram- allergic reaction ( } 1^{\text {st }} \\
\text { dose })\end{array}$ \\
\hline $\begin{array}{l}\text { Time since } \\
\text { Rituximab } \\
\text { (days/months) }\end{array}$ & 9 months & 55 months & 4 days \\
\hline $\begin{array}{l}\text { Indication organ } \\
\text { involvement at } \\
\text { this presentation }\end{array}$ & $\begin{array}{c}\text { Neurological (headaches, } \\
\text { memory loss, behavioural } \\
\text { change, non-specific } \\
\text { white matter changes on } \\
\text { MRI Brain) } \\
\text { Haematological } \\
\text { (Anaemia) } \\
\text { Renal } \\
\text { (Lupus nephritis, ISN/RPS } \\
\text { Class III) }\end{array}$ & $\begin{array}{c}\text { Renal } \\
\text { Lupus nephritis, ISN/RPS } \\
\text { Class III) } \\
\text { Serositis (pleural and } \\
\text { pericardial) } \\
\text { Haematological } \\
\text { (leukopenia, neutropenia, } \\
\text { lymphopenia) } \\
\text { Arthritis } \\
\text { Chronic cutaneous lupus } \\
\text { Non-scarring alopecia }\end{array}$ & $\begin{array}{l}\text { Haematological } \\
\text { (ITP, anaemia) } \\
\text { Arthritis }\end{array}$ \\
\hline $\begin{array}{l}\text { Cumulative } \\
\text { Ofatumumab } \\
\text { dose, IV, mg }\end{array}$ & $\begin{array}{l}2 \times 700 \mathrm{mg} \\
(1.4 \text { gram })\end{array}$ & $\begin{array}{l}4 \times 700 \mathrm{mg} \\
(2.8 \mathrm{gram})\end{array}$ & $\begin{array}{l}2 \times 700 \mathrm{mg} \\
(1.4 \text { gram })\end{array}$ \\
\hline $\begin{array}{l}\text { Number of } \\
\text { Ofatumumab } \\
\text { course }\end{array}$ & 1 course & 2 courses & 1 course \\
\hline $\begin{array}{l}\text { Maintenance } \\
\text { Therapy post- } \\
\text { Ofatumumab }\end{array}$ & $\mathrm{HCQ}, \mathrm{MMF}$, prednisolone & $\mathrm{HCQ}, \mathrm{MMF}$ & $\mathrm{HCQ}, \mathrm{MMF}$ \\
\hline ANA & $\begin{array}{l}\text { Homogeneous N/A } \\
>1: 2560\end{array}$ & $\begin{array}{l}\text { Speckled } \\
>1: 2560\end{array}$ & $\begin{array}{ll}\text { Speckled } & \text { Speckled } \\
>1: 2560 & >1: 2560\end{array}$ \\
\hline dsDNA & 318.0 & 156 & 1.8 \\
\hline ENA & Negative & $\begin{array}{l}\text { Positive: Anti-RNP, Ro, La, } \\
\text { Sm } \\
\text { Ribosomal P IgG Ab }\end{array}$ & Positive: Anti-Ro \\
\hline
\end{tabular}




\begin{tabular}{|c|c|c|c|c|c|c|}
\hline $\begin{array}{l}\text { Rheumatoid } \\
\text { Factor } \\
\text { (RR: 0-14 IU/ml) }\end{array}$ & \multicolumn{2}{|c|}{$\begin{array}{l}\text { Negative } \\
<10.0\end{array}$} & \multicolumn{2}{|c|}{$\begin{array}{l}\text { Negative } \\
<10.0\end{array}$} & \multicolumn{2}{|c|}{$\begin{array}{c}\text { Positive } \\
24.0\end{array}$} \\
\hline $\begin{array}{l}\text { Anti-C1q } \\
\text { antibodies } \\
\text { (RR:0-15 U/ml) }\end{array}$ & \multicolumn{2}{|c|}{ N/A } & 376.0 & 260.0 & \multicolumn{2}{|c|}{$<6.0$} \\
\hline DAT (Coombs) & \multicolumn{2}{|c|}{ Negative } & \multicolumn{2}{|c|}{ Negative } & \multicolumn{2}{|c|}{ Positive } \\
\hline $\begin{array}{l}\text { Total lymphocyte } \\
\text { count } \\
(1.20-5.20 \\
\text { X10*9/L) }\end{array}$ & 1.05 & 0.96 & 0.63 & 0.41 & 6.23 & 1.81 \\
\hline $\begin{array}{l}\text { Complement } 3 \\
\text { (RR:0.75-1.65 } \\
\text { g/l) }\end{array}$ & 0.61 & 0.89 & 0.44 & 0.63 & 1.64 & 1.79 \\
\hline $\begin{array}{l}\text { Complement } 4 \\
\text { (RR:0.14-0.54 } \\
\text { g/l) }\end{array}$ & 0.09 & 0.20 & 0.06 & 0.08 & 0.09 & 0.41 \\
\hline $\begin{array}{l}\text { WCC } \\
(4.00-11.00 \\
\times 10 * 9 / \mathrm{L})\end{array}$ & 2.73 & 3.89 & 2.40 & 1.88 & 19.66 & 5.09 \\
\hline $\begin{array}{l}\text { Neutrophils } \\
(1.80-8.00 \\
\times 10 * 9 / \mathrm{L})\end{array}$ & 1.36 & 2.37 & 1.46 & 1.22 & 12.01 & 2.89 \\
\hline $\begin{array}{l}\text { Platelets } \\
(150-450 \\
X 10 * 9 / L)\end{array}$ & 165 & 511 & 255 & 252 & 41 & 249 \\
\hline $\begin{array}{l}\text { Haemoglobin } \\
\text { (RR: } 120-160 \mathrm{~g} / \mathrm{L} \text { ) }\end{array}$ & 88 & 73 & 105 & 95 & 96 & 133 \\
\hline $\begin{array}{l}\text { IgG (RR: 5.4-16.1 } \\
\text { g/L) }\end{array}$ & 10.30 & 6.37 & 20.20 & 19.50 & 33.70 & 10.30 \\
\hline $\begin{array}{l}\operatorname{lgA} \\
\text { (RR: 0.7-2.5 g/L) }\end{array}$ & 1.18 & 0.81 & 2.71 & 3.38 & 1.45 & 1.24 \\
\hline $\begin{array}{l}\text { IgM } \\
\text { (RR: } 0.5-1.8 \mathrm{~g} / \mathrm{L})\end{array}$ & 0.61 & 0.51 & 1.02 & 1.33 & 0.80 & 0.47 \\
\hline $\begin{array}{l}\text { Urea } \\
\text { Mmol/L }\end{array}$ & 5.6 & 3.2 & 2.9 & 2.6 & 4.3 & 3.3 \\
\hline $\begin{array}{l}\text { Creatinine } \\
\mathrm{Mmol} / \mathrm{L}\end{array}$ & 71 & 39 & 46 & 39 & 43 & 46 \\
\hline $\begin{array}{l}\text { Albumin } \\
\text { g/L } \\
\text { (RR: } 37-56 \mathrm{~g} / \mathrm{L} \text { ) }\end{array}$ & 31 & 33 & 39 & 37 & 42 & 44 \\
\hline
\end{tabular}




\begin{tabular}{|c|c|c|c|c|c|c|}
\hline $\begin{array}{l}\text { Urine Alb/Cr } \\
\text { ratio } \\
\text { (RR: 0.7-7.4 } \\
\mathrm{mg} / \mathrm{mmol} \text { ) }\end{array}$ & 561.9 & 329.2 & 1.4 & 3.7 & 2.0 & 1.1 \\
\hline SLE-DAI & 28 & 2 & 40 & 11 & 25 & 4 \\
\hline BILAG-2004 & $A$ & $D$ & $B$ & D & $A$ & D \\
\hline $\begin{array}{l}\text { B cell } \\
\text { repopulation } \\
\text { after } \\
\text { Ofatumumab } \\
\text { (months) }\end{array}$ & \multicolumn{2}{|c|}{ N/A } & \multicolumn{2}{|c|}{ ABS CD19: 0.02} & \multicolumn{2}{|c|}{ ABS CD19: 0.28} \\
\hline
\end{tabular}

IVMP: Intravenous methylprednisolone, HCQ: Hydroxychloroquine, MMF: Mycophenolate mofetil, CYP: Cyclophosphamide, AZA: Azathioprine IVIg: Intravenous immunoglobulin

Table 1. Demographics, clinical, laboratory and treatment characteristics of the cases 